\title{
Etude morphodynamique du domaine pré-littoral nord aquitain, site atelier du Truc-Vert
}

\author{
Franck DESMAZES*, Denis MICHEL*, Hélène HOWA** et \\ Rodrigo PEDREROS*
}

*Département de Géologie et Océanographie, UMR CNRS EPOC 5805, Université Bordeaux I, Avenue des facultés, 33405 Talence cedex

e-mail : fdesmazes@epoc.u-bordeaux.fr; tel : 05-56-84-84-38

**Université d'Angers - UFR Sciences, 2 Bld Lavoisier, 49045 Angers Cedex

Résumé

La plage sous-marine nord-aquitaine est caractérisée dans sa partie supérieure (de 0 à $-11 \mathrm{~m}$ ) par un système de barre sableuse festonnée et de fosse pré-littorale, qui sont mobiles à l'échelle d'un an. Sa partie inférieure est plus stable, elle est formée d'une pente de $1 \%$ s'amortissant autour de $22 \mathrm{~m}$ de profondeur, à la frontière $\mathrm{du}$ plateau continental interne. Les limites morphologiques et morphodynamiques des deux domaines de la plage sous-marine se corrèlent très bien avec les paramètres de profondeur de fermeture (de $-10,8$ à $-12,8 \mathrm{~m}$ ) et de limite d'action des vagues ($23 \mathrm{~m}$ ). Cette relation remarquable est le signe d'un domaine très sensible aux conditions hydrodynamiques, et notamment aux tempêtes

\section{Abstract}

The north aquitanian upper shoreface $(0$ to $-11 \mathrm{~m})$ is composed by a dynamic bar and trough system, at the annual scale. The slope of the lower shoreface is $1 \%$ until -22 $m$ where inner continental shelf takes place. Morphological and morphodynamic limits are linked with closure depth and wave base formulation. This result is the sign of a high sensibility to hydrodynamics conditions, and to storm that will be further investigated.

\section{Introduction}

Le domaine pré-littoral se définie comme la transition entre le domaine intertidal et le plateau continental interne (Cacchione et Drake, 1990). Dans le cas de côtes sableuses, cette zone est aussi nommée plage sous-marine ou subtidale. C'est une zone complexe, siège de nombreux phénomènes hydro-sédimentaires liés à la transformation à la côte de la marée, des ondes gravitaires, des courants induits et des vents. Sa morphologie est contrôlée par de nombreux facteurs comme les vagues, les apports sédimentaires, les tempêtes, la variation du niveau marin. Ces facteurs, agissant sur des échelles de temps et d'espace très différentes, rendent complexe toute étude de cet environnement.

Les formes sédimentaires les plus caractéristiques des plages sous-marines sont les barres sableuses. De nombreux auteurs ont décrit ces barres qui peuvent être simples, doubles (Holman et Sallenger, 1993), multiples, rectilignes (Davis et Fox, 1972), festonnées (Goldsmith et al., 1982), transverses (Komar, 1998). Leur 
fonctionnement est déterminant pour le domaine littoral dans son ensemble car elles sont au cœur des échanges sédimentaires de la plage au sens large : elles forment des stocks sableux mobiles et peuvent jouer un rôle de protection de la côte lors des tempêtes (Lippmann et Holman, 1990).

Cet article présente les résultats des premières missions océanographiques menées sur la plage sous-marine nord-aquitaine. Ces campagnes de mesures, organisées dans le cadre de l'Action de Recherche "Hydrodynamique sédimentaire" du Programme National Environnement Côtier, ont permis de cartographier une partie de la plage sous-marine, d'effectuer des enregistrements des paramètres hydrodynamiques ainsi que des prélèvements de sédiments par carottage. Dans un premier temps, ces données vont nous permettre de caractériser la morphologie de la zone, puis nous nous intéresserons aux changements morphodynamiques avant d'étudier la sédimentation résiduelle de la plage sousmarine nord-aquitaine.

\section{Zone d'étude}

Le littoral nord-aquitain est une côte basse rectiligne et sableuse. Il s'étend sur $100 \mathrm{~km}$ selon une direction $\mathrm{N}^{\circ}$, de l'embouchure de la Gironde, au nord, à l'embouchure d'Arcachon, au sud. La plage sous-marine reste une zone peu étudiée car le contexte hydrodynamique complique les mesures et les observations. En effet les statistiques effectuées par Penin (1980) et les sorties du modèle numérique Vagatla de Météo France montrent qu'au large, les houles moyennes ont une hauteur significative $(\mathrm{Hs})$ de $2 \mathrm{~m}$ pour une période significative (Ts) de $8 \mathrm{~s}$.

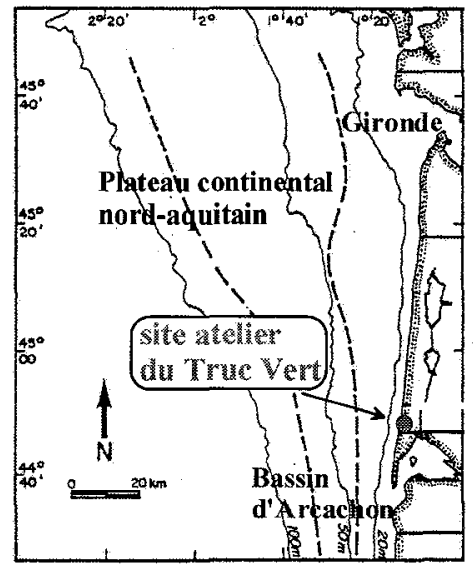

Figure 1 : zone d'étude Figure 1 :Study area

Ces valeurs sont fréquemment dépassées durant les coups de vent et atteignent alors $3,5 \mathrm{~m}$ durant $10 \%$ du temps. Les tempêtes les plus violentes peuvent, quant à elles, lever des houles de 4 à $6 \mathrm{~m}$ de hauteur significative. La marée est de type méso à macro tidal, son marnage varie de $1,5 \mathrm{~m}$ en morte-eau à $5 \mathrm{~m}$ en vive-eau.

Les missions océanographiques Phes 99 et Prislit 2000 et les campagnes de mesures PNOC 98 et PNEC 99 ont permis l'investigation précise d'un site atelier particulièrement représentatif de la côte nord-aquitaine : la plage du Truc Vert. En condition de beau temps, elle est caractérisée par :

- Une plage aérienne limitée par une dune littorale et une berme (Pedreros, 2000).

- Une plage intertidale, formée d'un système rythmique de barres et baïnes ("ridge and runnel") de $400 \mathrm{~m}$ de longueur d'onde (Michel et Howa, 1999).

- Une plage sous-marine, objet de cette étude, située sous le niveau des plus basses mers (Desmazes et al., 2001). 
3. Morphologie de la plage sous-marine nord-aquitaine (Figure 2)

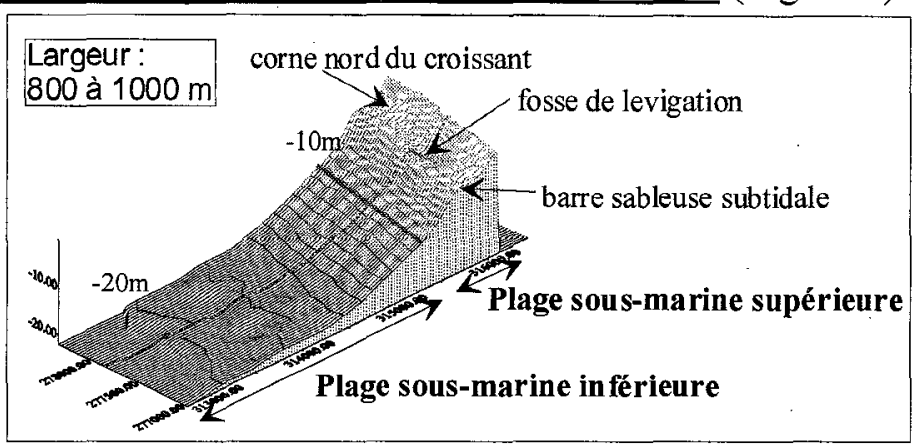

Figure 2 : Morphologie de la plage sous-marine nord-aquitaine Figure 2 : Morphology of the north-aquitanian shoreface

Sur la côte nord aquitaine, la plage sous-marine (ou subtidale) est constituée de deux entités morphologiques bien distinctes.

\subsection{La plage sous-marine supérieure}

Elle présente un système de barre festonnée séparée de la côte par une fosse pré-littorale (Froidefond et al., 1990). La barre sableuse est une structure rythmique et complexe, constituée de croissants d'une longueur d'onde moyenne de $1000 \mathrm{~m}$. Les cornes des croissants viennent plus ou moins s'accoler à la côte sous une profondeur d'eau de $2 \mathrm{~m}$. Entre ces cornes, on trouve une fosse prélittorale qui peut atteindre $7 \mathrm{~m}$ de profondeur. Le milieu du croissant se trouve à $450 \mathrm{~m}$ de la côte, sous 4,5m d'eau. A environ $11 \mathrm{~m}$ sous le zéro marin, une rupture de pente nette indique la limite morphologique externe de la barre pré-littorale et de la plage sous-marine supérieure.

\subsection{La plage sous-marine inférieure}

Au large de la rupture de pente à $-11 \mathrm{~m}$, la plage sous-marine inférieure présente une pente de $1 \%$ se développant sur $1 \mathrm{~km}$. Une nouvelle rupture de pente, située à environ $22 \mathrm{~m}$ sous le zéro marin, marque la frontière morphologique de la plage sous-marine et du plateau continental interne qui montre une pente moyenne de $0,25 \%$.

\section{Morphodynamique}

\subsection{Morphodynamique de la barre sableuse pré-littorale (Figure 3)}

La figure 3 montre l'évolution d'une corne de croissant entre $+5 \mathrm{~m}$ et $-7 \mathrm{~m}$, sur une largeur de plage de $200 \mathrm{~m}$. Le profil de mai 1998 montre la présence d'un système de barre et de fosse pré-littorales bien marqué, concomitant avec une barre intertidale bien développée. Cette morphologie s'est mise en place en réponse à des conditions hydrodynamiques modérées. Le profil d'avril 1999 est très clairement dissipatif, reflétant le lissage du profil et la redistribution du sable sur l'ensemble de la plage, sous l'influence des fortes conditions hydrodynamiques précédant les mesures. Les calculs de volume nous montrent 
qu'entre mai 1998 et avril 1999, la plage intertidale et le haut de plage se sont érodés de $40000 \mathrm{~m}^{3}$ tandis que la plage sous-marine supérieure présente une accrétion de $100000 \mathrm{~m}^{3}$. Ces données prouvent i) que la barre sableuse prélittorale se modifie notablement à l'échelle annuelle; et ii) qu'elle répond avec une forte sensibilité aux évènements météorologiques. Le degré de sensibilité de réponse et sa quantification sont à l'étude actuellement. D'autre part, les fonctionnements de la plage subtidale et de la plage intertidale sont très fortement liés par des échanges de sable qui interviennent en fonction des conditions hydrodynamiques.
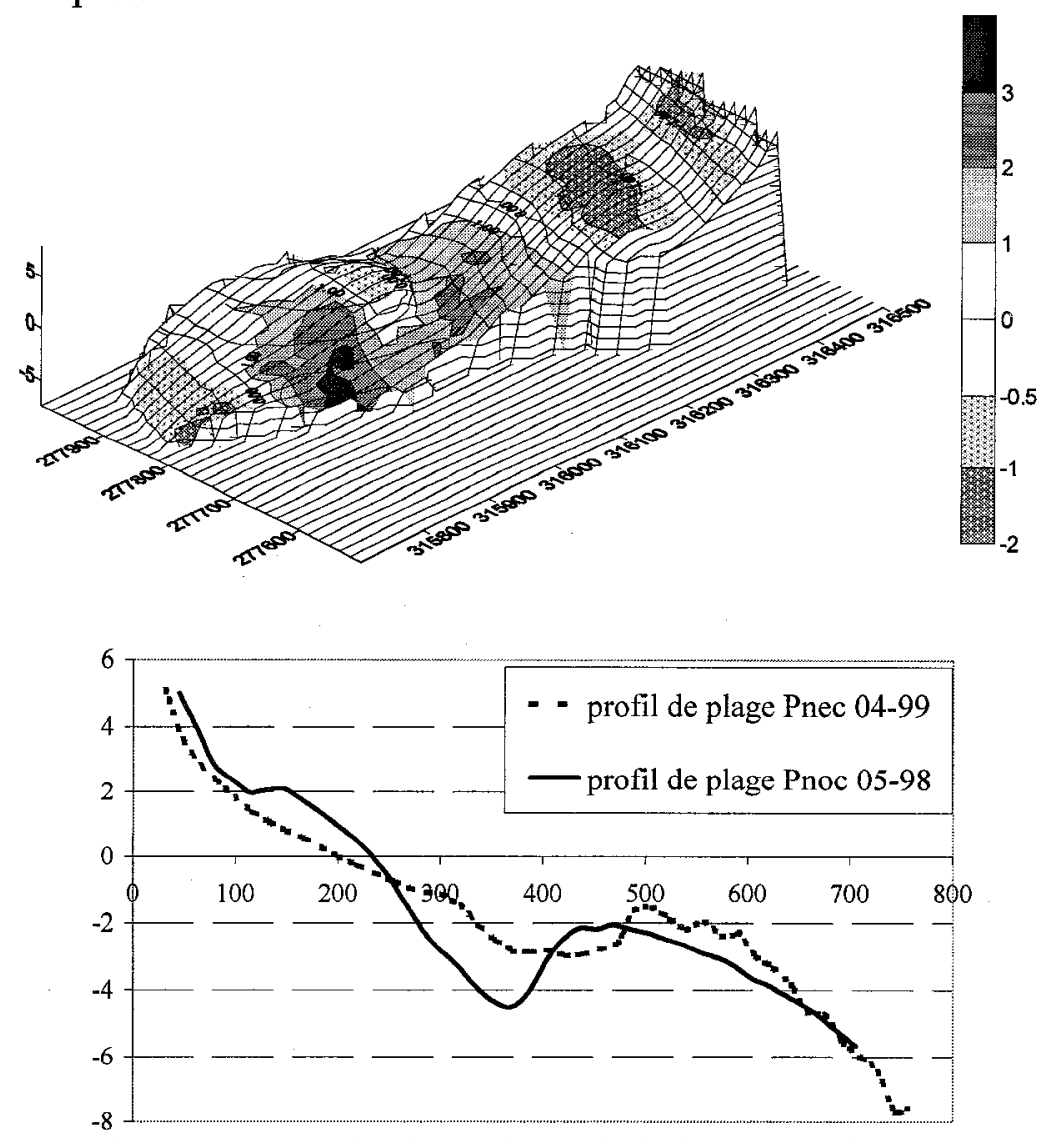

Figure 3 : Morphodynamique de la barre pré-littorale

Figure 3 : Morphodynamic of submarin sand bar

\subsection{Morphodynamique de la plage sous-marine inférieure (Figure 4)}

Les profils effectués entre -7 et $-21 \mathrm{~m}$ confirment la grande mobilité de la barre, à l'échelle inter-annuelle. On enregistre ainsi une érosion de $-2,8 \mathrm{~m}$ entre 1967 et 2000, à $10 \mathrm{~m}$ de profondeur. Entre -11 et $-20 \mathrm{~m}$ par contre, les variations de bathymétrie ne dépassent pas $0,5 \mathrm{~m}$. Enfin, au-delà de $-20 \mathrm{~m}$ de profondeur, on ne distingue de différence supérieure à $0,3 \mathrm{~m}$ (limite de résolution des sondages). Malgré cette incertitude, on peut faire l'hypothèse que la zone du plateau interne est morphologiquement stable à moyen terme, en comparaison avec la plage sousmarine. 


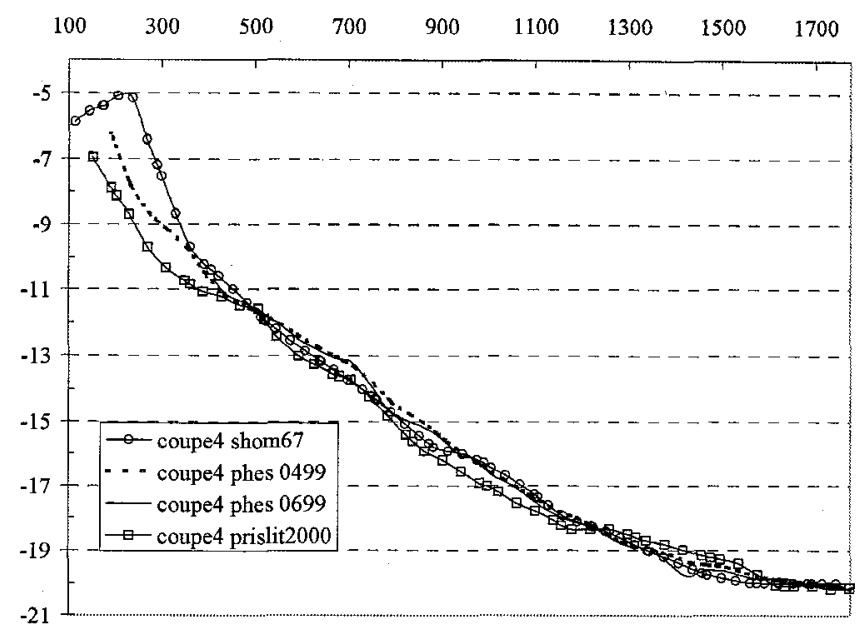

Figure 4 : Morphodynamique de la plage sous-marine inférieure

Figure 4: Morphodynamic of lower shoreface

\section{Sédimentation de la plage sous-marine (Figure 5)}

Le vibrocarottage VK06 a été prélevé à $10 \mathrm{~m}$ de profondeur. Les 80 premiers centimètres nous montrent l'alternance des faciès superficiels de sables moyens beiges et de sables fins gris. En dessous, on observe une séquence granodécroissante d'un mètre d'épaisseur. Sa base est constituée de sables très grossiers et de graviers, tandis que son sommet est formé de sables grossiers à moyens. Ce type d'enregistrement sédimentaire est caractéristique d'un événement extrêmement violent, il s'agit d'une tempestite proximale (Cirac et al., 2000). Une séquence d'une telle épaisseur implique que les tempêtes jouent un rôle fondamental dans la morphologie et la morphodynamique du domaine pré-littoral.

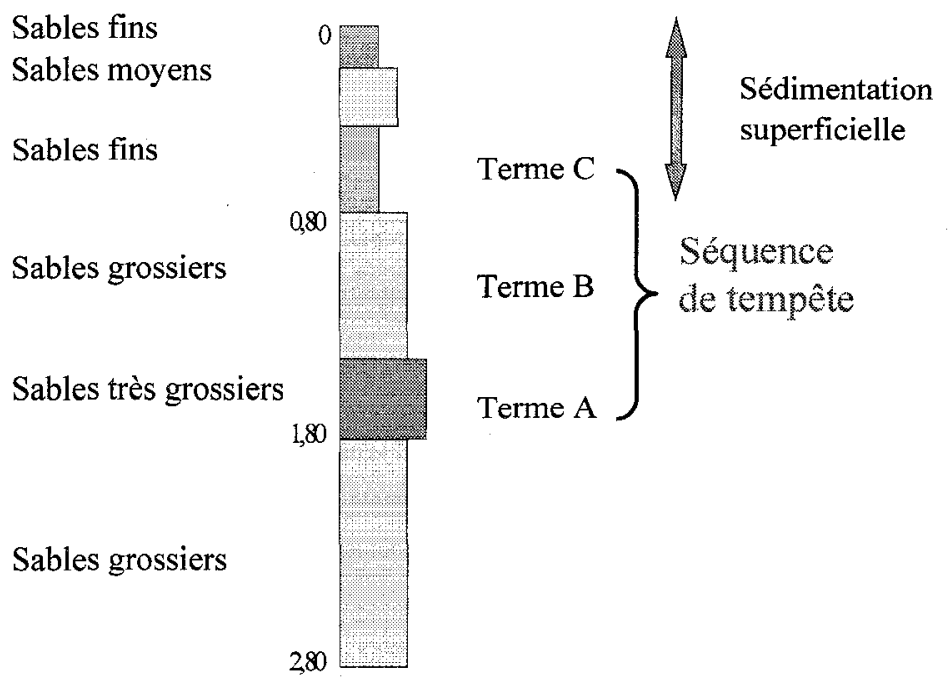

Figure 5 : Log synthétique de la carotte VK06 Figure 5 : Stratigraphical section of the core VK06 


\section{Discussion}

\subsection{Calcul des paramètres hydrodynamiques}

\subsubsection{Plage sous-marine supérieure}

Dans une approche hydrodynamique du domaine pré-littoral, Cowell et al. (1999) définissent la limite externe de la plage sous-marine inférieure par la notion de profondeur de fermeture $\left(\mathrm{h}_{\mathrm{c}}\right)$. La profondeur de fermeture correspond à la limite au-delà de laquelle on ne détecte plus de changement dans le profil, sur un an. Elle peut être estimée par une équation telle que :

$$
h_{c} \cong 2,28 \cdot H_{s x}-68,5 \cdot\left(\frac{H_{s x}^{2}}{g \cdot T_{e}^{2}}\right)
$$

Avec $\mathrm{H}_{\mathrm{sx}}$, hauteur des vagues de tempête qui n'est pas dépassée plus de $12 \mathrm{~h}$ par an ; $\mathrm{T}_{\mathrm{e}}$, période associée ; $\mathrm{g}$, accélération de la gravité.

Le calcul des profondeurs de fermeture entre 1998 et 2000 nous donne des valeurs comprises entre 10,8 et $12,8 \mathrm{~m}$ de profondeur. Ces indications sur la limite des variations annuelles du profil sont à mettre en relation avec la rupture de pente située à $-11 \mathrm{~m}(\S 3)$ ainsi qu'avec la limite de la zone mobile de barre pré-littorale à l'échelle inter-annuelle ( $-11 \mathrm{~m}$ également, $\S 4)$. Elles permettent de faire l'hypothèse d'une plage sous-marine supérieure et d'une barre pré-littorale qui sont mobiles à l'échelle annuelle et inter-annuelle, sous l'action des houles de tempêtes.

\subsubsection{Plage sous-marine inférieure}

Parmi un choix de formules simples (Hallermeier, 1981 ; Cowell et al., 1999), nous avons aussi pu relier les caractéristiques de la plage sous-marine inférieure aux conditions hydrodynamiques. La limite hydrodynamique externe de la plage sous-marine inférieure peut être définie par un paramètre de limite d'action des houles moyennes $\left(\mathrm{L}_{0} / 4\right)$ ne prenant pas en compte le seuil de déplacement des sédiments (Komar, 1998), tel que :

$$
L 0 / 4=\frac{g \cdot T^{2}}{8 \cdot \pi}
$$

avec $\mathrm{T}$, moyenne annuelle des périodes significatives des houles.

Son calcul nous donne une valeur d'environ $-23 \mathrm{~m}$, que l'on peut mettre en parallèle avec la rupture de pente séparant la plage sous-marine inférieure du plateau continental interne $(-22 \mathrm{~m}, \S 3.2)$. On peut aussi comparer ce paramètre à des mesures en nature par traçage radioactif (Migniot, 1977), ainsi qu'à la limite morphodynamique de la plage sous-marine inférieure $(-20 \mathrm{~m}, \S 4.2)$, sous-évaluée par la méthode bathymétrique. La corrélation des limites morphologiques et morphodynamiques avec le paramètre de limite d'action des houles moyennes de Komar permet de faire l'hypothèse d'une plage sous-marine inférieure dont la pente résiduelle serait une réponse morpho-sédimentaire à l'action des houles moyennes. 


\subsection{Action des tempêtes}

Il est probable que l'action continuelle des puissantes houles atlantiques sur les sédiments de surface soit à l'origine de la morphologie complexe du domaine pré-littoral aquitain. Néanmoins, il apparaît que l'action des tempêtes peut avoir un impact majeur à l'échelle d'une ou plusieurs années sur l'évolution de la barre pré-littorale et de l'ensemble de la zone ( $\$ 6.1 .1$ et Short, 1999). Les processus d'action des tempêtes sont encore mal connus sur la côte aquitaine. La structure des dépôts associés à ces évènements permet de penser qu'il s'agit soit d'un violent courant de retour du à une surcote de tempête, soit de courants de compensation générés par les vents qui établissent un système de circulation à deux couches, soit une combinaison de ces deux processus dynamiques (Reading, 1986).

Il semblerait en tout cas que l'impact de ces phénomènes peut être très important, comme en témoigne l'épaisseur du dépôt prélevé dans la carotte VK06 et les variations inter-annuelles des profils de plage sous-marine. De plus, les enregistrements de courant effectué à $10 \mathrm{~m}$ de profondeur pendant de fortes houles $(\mathrm{Hs}=5 \mathrm{~m}, \mathrm{Ts}=9 \mathrm{~s})$ ont permis d'observer des pics de vitesses orbitales dépassant $2 \mathrm{~m} . \mathrm{s}^{-1}$. Ce type de conditions provoque la mise en mouvement de la majorité des particules contenues dans le faciès très grossier à la base de la séquence de tempête (Hardisty, 1994). De la même manière, une extrême remobilisation des sédiments sur la plage intertidale et le haut de plage permet une érosion littorale importante. La mise en place de forts courants de compensation vers le large peut alors provoquer l'accrétion de la barre pré-littorale et de la plage sous-marine, induisant la translation vers le large de la profondeur de fermeture.

\section{Conclusions}

Les premières missions océanographiques menées dans le cadre du PNEC sur le domaine pré-littoral aquitain ont permis de mieux cerner la morphologie de cette zone et les caractéristiques de la barre sous-marine en croissant. Cette barre sableuse rythmique est très mobile d'une année sur l'autre, en fonction des conditions hydrodynamiques. La forte liaison de la morphologie et de 1'hydrodynamique de la zone a été mise en évidence par une très bonne corrélation des limites morphologiques, morphodynamiques et hydrodynamiques. La compréhension du système passe cependant par une étude en 3 dimensions sur une grande échelle spatiale et une modélisation des processus hydro-sédimentaires agissant sur la barre pré-littorale à court terme.

L'évolution de l'environnement à moyen terme (plusieurs années) dépend quant à lui des apports sédimentaires et surtout de la fréquence et de la force des tempêtes. Dans une perspective d'aménagement et de gestion du littoral aquitain, il semble important de mieux comprendre les mécanismes agissant lors de ces évènements exceptionnels. Mais il est surtout nécessaire de prévoir l'évolution du profil, son retour ou non à une position d'équilibre et les délais de reconstruction de la plage en fonction de l'impact de la tempête. 


\section{Références :}

Cacchione, D. A. and D. E. Drake (1990). Shelf sediment transport: an overview with applications to the northern California continental shelf. The Sea. Ocean Engineering Science. New York, Wiley. 9: 729-773.

Cirac, P., S. Berné, et al. (2000). Processus de mise en place et d'évolution de la couverture sédimentaire superficielle de la plate-forme nord-aquitaine. Océanologica Acta 23(6): 663-686.

Cowell, P. J., D. J. Hanslow, et al. (1999). The Shoreface. Handbook of Beach and Shoreface Morphodynamics. A. D. Short. New York, Wiley 39-71.

Davis, R. A., Jr and W. T. Fox (1972). Coastal processes and nearshore sand bars. Journal of Sedimentary petrology 42: 401-412.

Desmazes, F., D. Michel, et al. (2001). Etude morphologique et hydrosédimentaire du domaine pré-littoral aquitain. $8^{\mathrm{em}}$ Congrès Français de Sédimentologie, Orléans, ASF.

Froidefond, J. M., J. M. Gallissaires, et al. (1990). Spatial variation in sinusoidal wave energy on a crescentic nearshore bar ; application to the CapFerret Coast, France. J. C. R. 6(4): 927-942.

Goldsmith, V., D. Bowman, et al. (1982). Sequential Stage Development of Crescentic Bars : Hahoterim Beach. Jour. Sedim. Petrol. 34: 112-122.

Hallermeier, R.J. (1981). A profile zonation for seasonal sand beaches from wave climate, Coastal Engineering, 4, 253-277.

Hardisty, J. (1994). Beach and nearshore sediment transport. Sediment Transport and Depositional Processes. K. Pye. London, Blackwell : 219-255.

Holman, R. A. and J. Sallenger (1993). Sand Bar Generation : A Discussion of the Duck Experiment Series. J. C. R. 15(Special Issue): 76-92.

Komar, P. D. (1998). Beach processes and sedimentation. Prentice Hall.

Lippmann, T. C. and R. A. Holman (1990). The spatial and temporal variability of sand bar morphology. Jour. of Geophysic. Res. C7: 11575-11590.

Michel, D. and H. Howa (1999). Short term morphodynamic response of a ridge and runnel system on a mesotidal sandy beach. J. C. R. 15: 428-437.

Migniot, C. (1977). Action des courants, de la houle et du vent sur les sédiments. La Houille Blanche. 1. 10-47.

Pedreros, R. (2000). Quantification et modélisation du transport éolien au niveau des zones côtières- application au littoral girondin. Thèse, Bordeaux

Penin, F. (1980). Le prisme littoral aquitain : histoire holocène et évolution récente des environnements morphosédimentaires. Thèse, Bordeaux.

Reading, H.G. (1986). Sedimentary environments and facies, Blackwell.

Short, A. D. (1999). Handbook of beach and shoreface morphodynamics, Wiley. 